\title{
ChemComm
}

\section{Growth dynamics in supersonic molecular beam deposition of pentacene sub-monolayers on $\mathrm{SiO}_{2}$}

Cite this: Chem. Commun., 2014, 50,7694

Received 5th March 2014

Accepted 15th May 2014

DOI: $10.1039 / c 4 c c 01656 d$

www.rsc.org/chemcomm

\author{
Stefano Gottardi, ${ }^{a b}$ Tullio Toccoli, ${ }^{a}$ Yu Wu, ${ }^{b}$ Salvatore Iannotta ${ }^{a}$ and Petra Rudolf ${ }^{b}$
}

\begin{abstract}
Studying highly energetic pentacene impinging on a surface, we demonstrated that the perpendicular component of the momentum drives the dynamics of molecule-molecule interactions and hence the island nucleation process, while the parallel component governs the dynamics of the interactions between the surface and the molecule and therefore determines the sticking coefficient and the island fractality.
\end{abstract}

Pentacene is widely studied as a prototype molecule to understand the processes involved in charge and energy transport in molecular solids. ${ }^{1}$ To this end high quality films starting from the first layer formation are required. The first layer plays a crucial role not only as a template for the growth of the subsequent layers but also for charge transport; hence several reports focused on the study of the early growth stages in the sub-monolayer regime. ${ }^{2,3}$ Recently a new deposition technique based on supersonic molecular beams $(\mathrm{SuMBD})^{4}$ has been developed, which allows for improved control of the early stages of growth. With this technique the kinetic energy of impinging molecules can be easily tuned from thermal energy up to several $\mathrm{eV}$ by means of changing the degree of seeding in a lighter carrier gas ( $\mathrm{He}, \mathrm{Ar}$, etc.). ${ }^{4,5}$ Several reports appeared on the comparison between pentacene submonolayer morphologies for layers grown by SuMBD and by thermal sublimation $^{6}$ as well as on the carrier transport in field effect transistors based on SuMBD grown pentacene films. ${ }^{7}$ They show that the critical nucleus size, the crystallinity of the first monolayer and the structure of the molecular islands in the first pentacene layer are strongly correlated with the kinetic energy, $E_{\mathrm{k}}$, at which the molecules impinge on the substrate surface. However the mechanisms are not completely understood. A fundamental study of the interaction between energetic pentacene molecules and $\mathrm{a} \mathrm{SiO}_{x}$ surface by A. S. Killampalli et al. ${ }^{8}$ proposed that the decrease of pentacene adsorption probability with increasing

\footnotetext{
${ }^{a}$ IMEM-CNR, Istituto Materiali per Elettronica e Magnetismo,

Via alla Cascata 56/C, Povo Trento, Italy

${ }^{b}$ Zernike Institute for Advanced Materials, University of Groningen, Nijenborgh 4 ,

9747AG Groningen, The Netherlands. E-mail: p.rudolf@rug.nl
}

$E_{\mathrm{k}}$ is dominated by a trapping mediated process. ${ }^{9}$ The influence of parallel and normal momentum components of the impinging molecules was found to be equally important for adsorption under these conditions, as demonstrated by applying the energy scaling process in a regime between normal energy scaling ${ }^{10,11}$ and total energy scaling. ${ }^{12,13}$ However, so far no detailed study of the critical nucleus size, the variation of molecular island size and the evolution of sub-monolayer coverage for SuMBD growth at different $E_{\mathrm{k}}$ and momentum has been carried out. Here these relationships are revealed. We find that both the perpendicular and parallel momentum components of the molecules in the beam strongly influence the molecular sticking coefficient, the molecular island density, shape and size distribution as well as the critical nucleus size.

Pentacene was deposited under ultrahigh vacuum on a $500 \mathrm{~nm}$ thick $\mathrm{SiO}_{x} /$ silicon wafer (purchased from Silicon Quest International, USA) with a low root mean square surface roughness ( 5-6 $\AA$ ) as determined by atomic force microscopy in tapping mode (AFM). All substrates were moderately hydrophobic, with water contact angles of $55 \pm 2^{\circ}$ determined by the sessile drop method. Pentacene (Sigma-Aldrich, 99.98\%) was purified before use by gradient vacuum sublimation. The SuMBD system was described previously. ${ }^{5}$ Samples were prepared by exposure to the supersonic beam ( $\sim 10 \mathrm{~mm}$ diameter) at different incident angles, $\theta: 0^{\circ}$ (normal) incidence, $25^{\circ}, 45^{\circ}$ and $75^{\circ}$ (see the inset of Fig. 2), at room temperature. The molecular beam was characterized on-line in terms of chemical purity, flux, and energy distribution by combining time of flight (TOF) mass spectrometry and multiphoton ionization spectroscopy. We chose an operation regime where no clustering effects and contaminants were detectable. The typical flux was $6 \times 10^{11}$ molecules per $\left(\mathrm{s} \mathrm{cm}^{2}\right)$, estimated by cross correlating the TOF spectra at different $E_{\mathrm{k}}$ of the beam with that of a pentacene Knudsen source used as standard. The different $E_{\mathrm{k}}$ explored here were achieved by varying the degree of seeding by changing the He carrier gas pressure. Ex situ AFM was systematically carried out by scanning over multiple $10 \times 10 \mu \mathrm{m}^{2}$ areas at the sample centre (using Tap 300 probes, Budget sensor, on a Nanoscope IV multimode scanning probe microscope, 


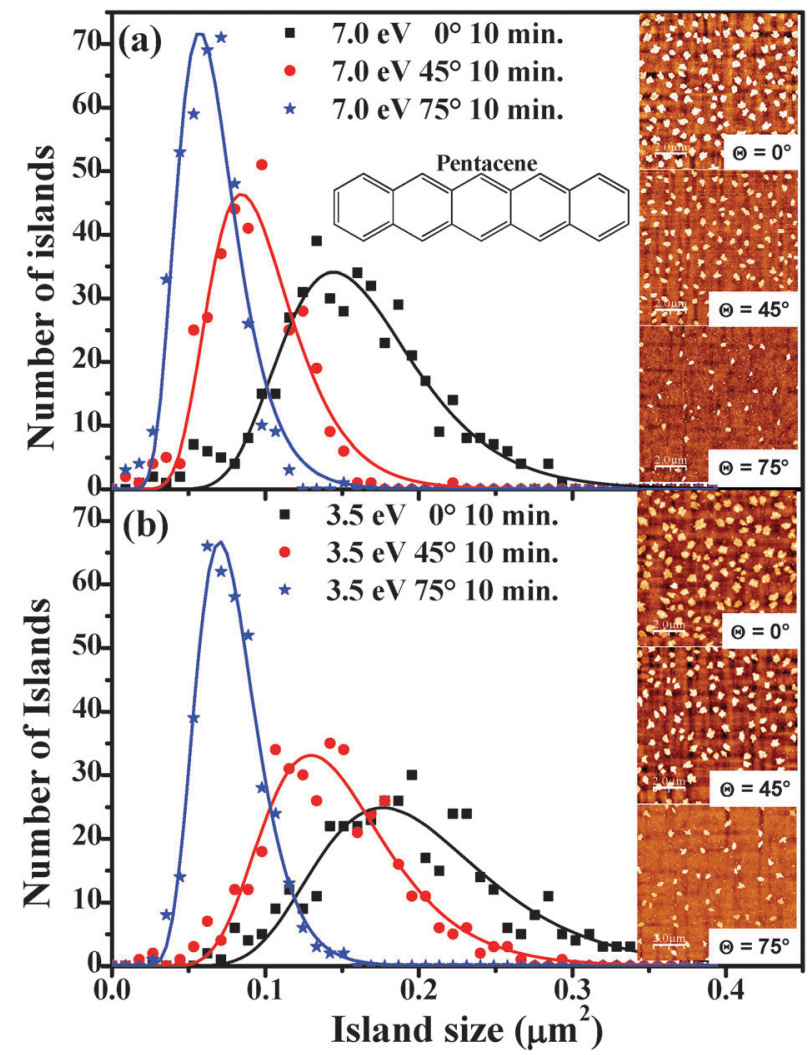

Fig. 1 Island size distribution in a pentacene sub-monolayer grown on $\mathrm{SiO}_{x}$ after 10 min of exposure to a supersonic beam of molecules with a kinetic energy of $7.0 \mathrm{eV}(\mathrm{a})$ and $3.5 \mathrm{eV}(\mathrm{b})$ at incidence angles $\theta=0,45^{\circ}$ and $75^{\circ}$ with respect to the surface normal. The insets show the typical island morphology as a function of incident angle $\theta$. In (a) the scheme of the pentacene molecule is also shown.

Digital Instruments, Veeco Metrology Group, U.S.A.). The analysis of these AFM images (with the help of the WSxM software, Nanotec electronics S.L.) provided the information on the influence of the momentum components of the impinging molecules on the growth processes such as the island size distribution, the critical nucleus size, the island shape and the sticking coefficient.

To gain insight into the growth dynamics, we considered samples obtained after $10 \mathrm{~min}$ exposure to the pentacene flux where the onset of island coalescence is not yet reached. The island size distribution and the average island size $\left(A_{\mathrm{av}}\right)$ for two different kinetic energies of the molecules $(7.0 \mathrm{eV}$ and $3.5 \mathrm{eV})$ impinging at three different incidence angles $\left(0^{\circ}, 45^{\circ}\right.$ and $\left.75^{\circ}\right)$ were extracted from the AFM images. AFM micrographs of the typical morphologies observed are shown in the inset of Fig. 1(a) and (b). At these early growth stages, the dependence of the morphology on the incident angle $\theta$ of the beam appears to be quite evident: both coverage and molecular island size decrease with increasing $\theta$, while the distance between islands increases. We observe also that the coverage decrease and the increase of the inter-island distance are much more evident for the higher kinetic energy. Practically the role of the momentum component parallel to the surface associated with $E_{\mathrm{k}}, M_{\|}$, becomes evident. The higher the $M_{\|}$, the larger is the

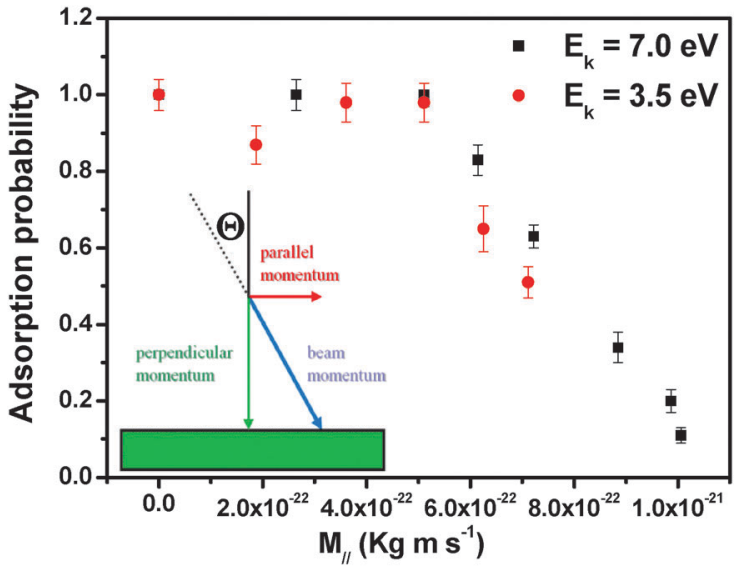

Fig. 2 Adsorption probability normalized to that for normal incidence growth (zero parallel momentum). The incidence angle $\theta$ varied from $0^{\circ}$ to $80^{\circ}$ with respect to the normal to the surface. The inset shows how the molecular momentum is divided into the $\|$ and $\perp$ components.

probability for the molecules to be scattered out from the surface plane, so that not only the overall amount of deposited molecules reduces (reduced sticking) but also the density and size of islands decrease. These can be quantified in the island size distribution shown in Fig. 1(a) and (b) where the histograms of the island size $\left(A_{\mathrm{av}}\right)$ under different growth conditions as obtained from AFM images collected over 5 scanning areas of $10 \times 10 \mu \mathrm{m}^{2}$ for each case are reported. The histograms were fitted using a $\log$ normal function ${ }^{6,18}$ with a single peak to determine the average island size. The obtained average island sizes were found to be $0.15 \pm 0.02,0.09 \pm 0.01$ and $0.06 \pm$ $0.01 \mu \mathrm{m}^{2}$ for samples grown at $E_{\mathrm{k}}=7.0 \mathrm{eV}$, at normal incidence, $\theta=45^{\circ}$ and $75^{\circ}$; the corresponding values for $E_{\mathrm{k}}=3.5 \mathrm{eV}$ amounted to $0.19 \pm 0.03,0.14 \pm 0.02$ and $0.07 \pm 0.01 \mu \mathrm{m}^{2}$ for normal incidence, $\theta=45^{\circ}$ and $75^{\circ}$. We observe that the average island size decreases with increasing $\theta$ and for each incidence angle, while the average island size for samples grown with $E_{\mathrm{k}}=3.5 \mathrm{eV}$ is always larger than the corresponding one for samples grown with $E_{\mathrm{k}}=7.0 \mathrm{eV}$. This suggests that molecules which impinge with smaller $E_{\mathrm{k}}$ experience less molecule-surface scattering and lower surface diffusion, which in turn favours the lateral expansion of the islands via trapping of more molecules on the surface. With increasing $\theta$, the distribution of island sizes obtained for the growth becomes narrower for both the kinetic energies used. The full width at half maximum of the peak drops from $0.10 \mu \mathrm{m}^{2}\left(0.12 \mu \mathrm{m}^{2}\right)$ at normal incidence to $0.06 \mu \mathrm{m}^{2}$ $\left(0.10 \mu \mathrm{m}^{2}\right)$ at $\theta=45^{\circ}$ and to $0.05 \mu \mathrm{m}^{2}\left(0.05 \mu \mathrm{m}^{2}\right)$ at $\theta=75^{\circ}$ for samples grown with $E_{\mathrm{k}}=7.0 \mathrm{eV}(3.5 \mathrm{eV})$. This indicates that the island size is more uniform when the molecules impinge at a larger incidence angle.

To quantify this effect we investigated the adsorption probability under different growth conditions. To compare the results obtained using different growth parameters, a surface coverage of $20 \%$ of a monolayer was chosen since at this stage of the growth the islands are big enough to show characteristic features but far away from coalescence. The flux of impinging molecules was adjusted to be constant at all angles of incidence. Moreover, we assumed a 
constant growth rate during the growth, which is a good assumption under our operation conditions. By comparing the deposition times that are needed to reach $20 \%$ coverage, one can obtain the adsorption probability which has been normalized to 1 for the reference samples grown with molecules impinging at normal incidence.

Fig. 2 shows the adsorption probability, as a function of the parallel momentum $\left(M_{\|}\right)$of the incident molecules. Two different adsorption regimes are clearly distinguished: for low values of $M_{\|}$ the adsorption probability is constant, while when the parallel momentum increases beyond a certain critical value $\left(M_{\|} \sim 5 \times\right.$ $10^{-22} \mathrm{~kg} \mathrm{~m} \mathrm{~s}{ }^{-1}$ ) the adsorption probability decreases linearly with $M_{\|}$. This is due to the difficulty in dissipating parallel momentum at the surface. The constant adsorption probability at lower $M_{\|}$is likely due to the fact that when a strong adsorption site becomes available, the influence of the parallel momentum on the adsorption probability is suppressed. In other words, desorption of the molecules can be activated only for sufficiently large values of $M_{\| \bullet}$. The barrier height cannot be deduced directly in our experiments; a comparison of these data with simulations would be interesting.

The observed dependence of the adsorption probability on $M_{\|}$is an indication of trapping processes involving possibly not only one step, as in direct trapping. Such a decrease of adsorption probability with increasing $\theta$ has been observed in very few cases, mostly when trapping occurs via dissipation of $E_{\mathrm{k}}$ associated with the normal component of momentum (normal energy scaling), $M_{\perp}$, as often true for simple (diatomic) molecules on surfaces of transition metals. ${ }^{14}$ Most of the cases where normal energy scaling is not observed have been interpreted in terms of (i) strong corrugation effects either of the potential energy surface or of the physical substrate surface, (ii) the role of internal degrees of freedom and/or (iii) inefficient accommodation of parallel momentum. ${ }^{9}$

A simple experimental way to quantify the relative role played by the perpendicular and parallel components of momentum linked to a specific kinetic energy of the impinging molecules is to introduce the empirical scaling function of the form $E_{\mathrm{K}} f(\theta)=E_{\mathrm{K}}\left(A \cos ^{2} \theta+B \sin ^{2} \theta\right)$ where $A+B=1$ and the energy associated with the perpendicular (parallel) momentum scales with coefficient $A(B) .{ }^{15}$ Following this procedure for our data, we find values for $A$ and $B$ which, within the error bars, are identical to those found by A. S. Killampalli et al. ${ }^{8}$ However, we do not arrive at their conclusion that the dissipation of the parallel and the perpendicular momentum component is roughly equally important.

In fact, an understanding of these trends may be achieved by comparing with classical molecular dynamics simulations for SuMBD of ethane on $\operatorname{Si}(100),{ }^{13}$ a system where a similar sticking behaviour was observed experimentally. These simulations show that the energy exchanged upon the first impact on the surface largely dictates whether the incident molecule will stay on the surface or not. During this first collision the energy associated with the normal momentum component is dissipated very effectively, whereas that associated with the parallel momentum component is not. This process leaves the molecule in a vibrationally and rotationally excited state which allows it to make subsequent impacts on the surface and undergo further energy dissipation processes. However, molecules with a large parallel momentum component were found to retain it in large part after the initial impact on the surface. ${ }^{9}$ Parallel momentum, as well as any energy stored in rotations, can be converted into normal momentum during subsequent impacts, causing the molecules to scatter back into the vacuum. Therefore, molecules with high kinetic energy impinging at glancing angles have a much larger probability of being scattered from the surface before the parallel momentum can be dissipated. Our data on pentacene where the sticking coefficient becomes smaller with larger incident angle and $E_{k}$ strongly support this mechanism.

The critical nucleus $i(i+1=$ number of molecules forming a stable nucleus) determined under the different growth conditions is an important criterion to gather information on the effect of momentum and kinetic energy on the molecular assembly processes. $i$ is determined from the general scaling function

$$
f_{i}(u)=C_{i} u^{i} \mathrm{e}^{-i a_{i} u^{\frac{1}{a_{i}}}}
$$

introduced by Amar and Family, ${ }^{16}$ extended to pentacene growth by Ruiz et al., ${ }^{17}$ and reported also by Tejima et al. ${ }^{18}$ and Stadlober et $a l^{19} C_{i}$ and $a_{i}$ are constants determined by hyper-geometrical equations for $i=0, \ldots, 3$ that ensure normalization and proper asymptotic behaviour of $f_{i}(u)$ - for details we refer the reader to ref. 16-19. We compared the normalized island size distributions of films grown under different conditions with the predictions of the general scaling model calculated for $i=1,2$, and 3. For samples grown with $E_{\mathrm{k}}=3.5 \mathrm{eV}, i=3$ for all incidence angles, while for those grown with $E_{\mathrm{k}}=7.0 \mathrm{eV}$, at normal incidence, $i=2$ as found previously, ${ }^{4}$ but for $\theta=45^{\circ}$ and $75^{\circ}, i=3$. This indicates that it is the energy associated with the normal momentum component, which determines the critical nucleus size and not the total $E_{\mathrm{k}}$. In fact, the normal energy components of molecules impinging with $E_{\mathrm{k}}=7.0 \mathrm{eV}$ at $\theta=45^{\circ}$ and $\theta=75^{\circ}$ are 3.5 and $0.5 \mathrm{eV}$, respectively, and hence fall into the range $<5.5-6.0 \mathrm{eV}$, where we previously found $i=3$ at normal incidence. ${ }^{4}$ This seems to indicate that it is the momentum component normal to the surface which determines the critical nucleus size, possibly due to its highly efficient dissipation upon the first impact. The change in critical nucleus size observed here is analogous to findings of a previous study on pentacene submonolayer growth where thermally sublimed pentacene impinged on a substrate held at different temperatures, ${ }^{2,20}$ and where higher substrate temperature was found to lead to a high probability of pentacene desorption on the surface, resulting in a higher value of critical nucleus size.

We also investigated how kinetic energy and incidence angle of the impinging molecules influence the island shape. To this end we determined the fractal dimension under different growth conditions from the AFM micrographs. The island fractal dimension $\left(D_{\mathrm{f}}\right)$, calculated using the area-perimeter relationship, as proposed in ref. 21

$$
P=k A^{D \mathrm{f} / 2}
$$

where $P$ is the perimeter of the island, $A$ its area, $k$ a scaling constant and $D_{\mathrm{f}}$ the fractal dimension, gives important information about the molecular diffusion length and the assembly 


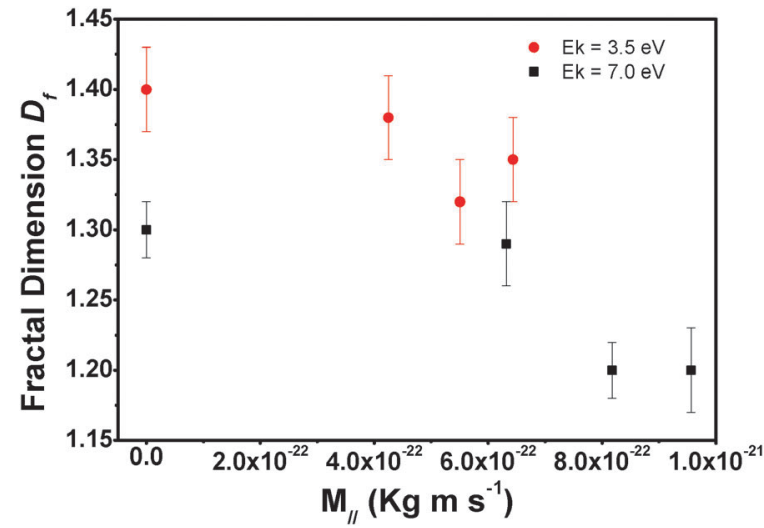

Fig. 3 Fractal dimension $\left(D_{f}\right)$ plotted as a function of the parallel momentum.

processes of the molecules. ${ }^{3}$ Under our deposition conditions, $D_{\mathrm{f}}$ scales with $\theta$ for all $E_{\mathrm{k}}$ : as the incident angle and/or the kinetic energy of the impinging molecules increase, the island fractal dimension decreases. These trends confirm that higher surface mobility of pentacene leads to more compact islands. To better understand whether a parallel or a perpendicular moment component plays the more important role, we analyzed the data as a function of $M_{\perp}$ and $M_{\|}$. The plot of the fractal dimension vs. $M_{\perp}$ does not show any particular dependence, indicating that this component has only a minimal role in the molecular surface diffusion, while $D_{\mathrm{f}} v s . M_{\|}$, reported in Fig. 3, shows a clear trend: the fractal dimension of the island shape decreases with increasing $M_{\|}$. We can therefore conclude that the momentum component parallel to the surface determines the island shape and size distribution.

In conclusion, we analysed the surface morphology of pentacene submonolayers grown by supersonic molecular beam deposition on $\mathrm{SiO}_{x} / \mathrm{Si}$ kept at room temperature. In particular, we focused on the influence of the kinetic energy of the impinging molecules and of the incidence angle. The observations can be rationalized in the following way: when pentacene collides with the $\mathrm{SiO}_{x}$ surface, it loses energy through different mechanisms which depend on its momentum and kinetic energy. In particular, $M_{\perp}$ is primarily linked to the formation of the initial nuclei: increasing this component we observed that fewer molecules were needed for the formation of stable nuclei. Higher $M_{\perp}$ also favours a higher number of islands and a decrease of inter-island distance. $M_{\|}$influences the interaction between the surface and molecules. Its dissipation modifies the sticking coefficient of the molecules and their relaxation processes, increasing their surface mobility. High values of $M_{\|}$ decrease the number of molecules on the surface but favour the formation of more compact (less fractal) islands, hence leading to better ordered films. $M_{\|}$influences the dynamics of moleculemolecule interactions on the surface while $M_{\perp}$ seems to be related to the dynamics of the coupling between molecules and surface phonons. To optimize the growth processes of organic molecules such as pentacene the right balance of both components is needed.

The authors would like to thank L. Venema, C. Corradi and M. Pola for technical assistance. We also thank Norbert Koch and Roberto Verucchi for precious discussions. This work was financially supported by the Dutch Foundation for Fundamental Research on Matter (FOM), and by the Breedtestrategie program of the University of Groningen.

\section{Notes and references}

1 R. Ruiz, D. Choudhary, B. Nickel, T. Toccoli, K. C. Chang, A. C. Mayer, P. Clancy, J. M. Blakely, R. L. Headrick, S. Iannotta and G. G. Malliaras, Chem. Mater., 2004, 16, 4497.

2 D. Choudhary, P. Clancy, R. Shetty and F. Escobedo, Adv. Funct. Mater., 2006, 16, 1768.

3 S. Pratontep, M. Brinkmann, F. Nuesch and L. Zuppiroli, Phys. Rev. B, 2004, 69, 165201.

4 Y. Wu, T. Toccoli, N. Koch, E. Iacob, A. Pallaoro, P. Rudolf and S. Iannotta, Phys. Rev. Lett., 2007, 98, 076601.

5 S. Iannotta and T. Toccoli, J. Polym. Sci., Part B: Polym. Phys., 2003, 41, 2501.

6 Y. Wu, T. Toccoli, J. Zhang, N. Koch, E. Iacob, A. Pallaoro, S. Iannotta and P. Rudolf, Appl. Phys. A: Mater. Sci. Process., 2009, 95, 21.

7 T. Toccoli, A. Pallaoro, N. Coppedè, S. Iannotta, F. D. Angelis, L. Mariucci and G. Fortunato, Appl. Phys. Lett., 2006, 88, 132106.

8 A. S. Killampalli, T. W. Schroeder and J. R. Engstrom, Appl. Phys. Lett., 2005, 87, 033110.

9 C. T. Reeves, B. A. Ferguson, C. B. Mullins, G. O. Sitz, B. A. Helmer and D. B. Graves, J. Chem. Phys., 1999, 111, 7567.

10 F. O. Goodman and H. Y. Wachman, Dynamics of Gas - Surface Scattering, Academic Press, New York, 1976.

11 J. A. Barker and D. J. Auerbach, Surf. Sci. Rep., 1984, 4, 1.

12 S. Andersson, L. Wilzen and J. Harris, Phys. Rev. Lett., 1986, 57, 1603.

13 C. T. Rettner, H. Stein and E. K. Schweizer, J. Chem. Phys., 1988, 89, 3337.

14 C. T. Rettner, D. J. Auerbach, J. C. Tully and A. W. Kleyn, J. Phys. Chem., 1996, 100, 13021.

15 M. C. McMaster, S. Schroeder and R. J. Madix, Surf. Sci., 1993, 297, 253.

16 J. G. Amar and F. Family, Phys. Rev. Lett., 1995, 74, 2066.

17 R. Ruiz, B. Nickel, N. Koch, L. C. Feldman, R. F. Haglund Jr., A. Kahn, F. Family and G. Scoles, Phys. Rev. Lett., 2003, 91, 136102.

18 M. Tejima, K. Kita, K. Kyuno and A. Toriumi, Appl. Phys. Lett., 2004, 85, 3746.

19 B. Stadlober, U. Haas, H. Maresch and A. Haase, Phys. Rev. B, 2006, 74, 165302.

20 A. C. Mayer, R. Ruiz, R. L. Headrick, A. Kazimirove and G. G. Malliaras, Org. Electron., 2004, 5, 257.

21 P. A. Burrough, Principles of Geographical Systems for Land Resources Assessment, Clarendon, Oxford, 1986. 